\title{
Uso de analizador de emociones en sistemas educativos inteligentes
}

\author{
María Lucia Barrón-Estrada ${ }^{1}$, Ramón Zatarain-Cabada ${ }^{1}$, \\ Sandra Lucia Ramírez-Ávila ${ }^{1}$, Raúl Oramas-Bustillos ${ }^{1}$, \\ Mario Graff Guerrero ${ }^{2}$ \\ ${ }^{1}$ Tecnológico Nacional de México, Instituto Tecnológico de Culiacán, \\ México \\ ${ }^{2}$ INFOTEC, Aguascalientes, \\ México \\ \{lbarron, rzatarain, sramirez, raul.oramas\}@itculiacan.edu.mx, \\ mario.graff@infotec.mx
}

\begin{abstract}
Resumen. En este trabajo se presenta el desarrollo de un clasificador de frases relacionadas con el aprendizaje en el ámbito de programación de computadoras para realizar análisis de sentimientos en sistemas educativos inteligentes. El clasificador se ofrece como un servicio web que recibe un texto en español y regresa como resultado una emoción centrada en el aprendizaje. El clasificador fue entrenado con un corpus de frases en español expresadas por estudiantes al acceder a diversos objetos de aprendizaje. El corpus se creó mediante el Sistema de Evaluación de Recursos Educativos (SERE) que se encarga de recolectar frases textuales escritas en español las cuales reflejan la opinión de los estudiantes sobre los recursos educativos utilizados en el aprendizaje de diversos temas. Las opiniones (frases textuales) fueron etiquetadas para categorizarlas en diferentes emociones centradas en el aprendizaje tales como: Frustrado, Aburrido, Emocionado y Comprometido. La principal contribución de este trabajo es un analizador para el reconocimiento de emociones centradas en el aprendizaje usando frases textuales escritas en español que podrá ser utilizado por un sistema tutor inteligente para detectar emociones de los estudiantes y realizar de forma más eficiente el proceso de enseñanza con los estudiantes adaptando el contenido tanto a las necesidades cognitivas como afectivas.
\end{abstract}

Palabras clave: análisis de texto, análisis de sentimientos, minería de opiniones, sistemas educativos inteligentes, entorno de aprendizaje inteligente, emociones en el aprendizaje.

\section{Use of Emotion Analyzer in Intelligent Educational Systems}

Abstract. In this paper, we present the development of a classifier of Spanish sentences related to learning in the field of computer programming to make sentiment analysis inside intelligent educational systems. The classifier is offered 


\begin{abstract}
as a web service that receives a text in Spanish and returns as a result a learning centered emotion. The classifier was trained with a corpus of Spanish phrases expressed by students after accessing different learning objects. The corpus was created through the Educational Resources Evaluation System (SERE) that is responsible for collecting textual phrases written in Spanish which reflect the students' opinion about the educational resources used in the learning of various topics. The opinions (textual phrases) were labeled to categorize them into different learning centered emotions such as: frustrated, bored, excited and engaged. The main contribution of this work is an analyzer for the recognition of learning centered emotions using textual phrases written in Spanish; which can be used by an intelligent tutor system to detect students' emotions and to execute more efficiently the teaching process with the students, and adapting the content to both cognitive and affective needs.
\end{abstract}

Keywords: text analysis, sentiment analysis, opinion mining, intelligent educational systems, intelligent learning environment, learning-centered emotions.

\title{
1. Introducción
}

En un ambiente educativo tradicional los estudiantes interactúan con sus compañeros para intercambiar experiencias y colaborar en el desarrollo de proyectos o la resolución de problemas con el fin de aprender diversos temas. En el modelo constructivista, el profesor es un facilitador que proporciona herramientas y guía a los estudiantes en el proceso de construcción de su propio conocimiento. Sin embargo, los programas de estudio no son personalizados y todos los estudiantes deben acceder a los mismos temas en los mismos tiempos. Además, las emociones juegan un papel fundamental durante el aprendizaje y pueden propiciar un mejor aprovechamiento o bloquear el aprendizaje, por eso es importante detectar las emociones y actuar acorde a éstas con el fin de mantener al estudiante interesado en el tema de estudio.

Se han desarrollado diferentes tecnologías para enfrentar la necesidad de integrar las emociones en el proceso de enseñanza-aprendizaje. Entre estas tecnologías se encuentran algunos Sistemas Tutores Inteligentes (ITS, por sus siglas en inglés) y Entornos de Aprendizaje Inteligentes (ILE, por sus siglas en inglés) los cuales fueron diseñados para capturar e identificar las emociones de los usuarios, pero la mayoría de estos sistemas convencionales funcionan solo con emociones básicas [1].

En trabajos anteriores se han estudiado las emociones básicas, entre las que se encuentran: la ira, la felicidad, la tristeza y el miedo; las cuales se expresan en diferentes situaciones de la vida cotidiana [2]. Sin embargo, hay otro tipo de emociones que emergen durante las actividades de aprendizaje profundo y son llamadas emociones centradas en el aprendizaje [3,4]; en éstas un estudiante puede sentirse: frustrado, aburrido, comprometido o emocionado. Las emociones centradas en el aprendizaje juegan un papel importante en los estudiantes, ya que afectan diferentes aspectos como mecanismos cognitivos y retención de información [5,6].

Actualmente la mayoría de las herramientas para el reconocimiento de emociones en texto trabaja con corpus de frases textuales generales escritas en inglés que se recolectaron de diferentes redes sociales, aunque Tellez, Jiménez, Graff, Moctezuma, Siordia, \& Villaseñor, utilizan frases en español cuyo objetivo es analizar 
exhaustivamente todas las combinaciones de las transformaciones de texto para descubrir características comunes [16]. En estos trabajos, las frases no son específicas para el área de educación (aprendizaje de programación de computadoras) lo que hace necesario desarrollar un corpus de frases textuales en español que contengan información acerca de las emociones que los estudiantes experimentan durante el proceso de aprendizaje. Es por esto que se decidió crear una base datos específica para realizar análisis de sentimientos en frases textuales en español sobre el tema de programación de computadoras.

En este trabajo, se describen los resultados generados por el clasificador de reconocimiento de emociones. Para entrenar al clasificador, se utilizó el corpus de frases textuales en español, generado por el Sistema de Evaluación de Recursos Educativos (SERE). SERE tiene como propósito mostrar a los estudiantes diversos objetos de aprendizaje (OA) solicitándole que escriba una opinión textual sobre el OA. El clasificador podrá ser utilizado para incorporar cambios y mejoras a los contenidos de los cursos y otros elementos didácticos en los ITS o ILE.

La principal contribución de este trabajo es, el desarrollo un clasificador de frases textuales en español para el reconocimiento de emociones centradas en el aprendizaje por medio de texto.

Este artículo se organiza de la siguiente forma: la sección 2 presenta los trabajos relacionados, la sección 3 expone el proceso para realizar el análisis de sentimientos. La sección 4 muestra los resultados de las pruebas del clasificador y finalmente en la sección 5 se presentan las conclusiones.

\section{Trabajos relacionados}

En la década pasada, la proliferación de sitios de internet donde los usuarios podían expresar sus opiniones generó la necesidad de procesar automáticamente estas opiniones para obtener información relevante que pudiera ser utilizada para la toma de decisiones, surgiendo el área de Minería de Opiniones. Análisis del sentimiento o Minería de opiniones (SA u OM por sus siglas en inglés respectivamente) se define como la tarea de encontrar las opiniones de los autores sobre entidades específicas [7]. Otra definición conocida para SA es el estudio computacional de las opiniones, actitudes y emociones de las personas hacia una entidad [8].

OM extrae y analiza la opinión de la gente sobre una entidad mientras que SA identifica el sentimiento expresado en un texto y luego lo analiza. Por lo tanto, el objetivo de SA es encontrar opiniones, identificar los sentimientos que expresan y luego clasificar su polaridad [8]. Medhat et al [8], propone tres niveles principales de clasificación en SA, los cuales son:

- Nivel de documento SA: considera a todo el documento como una unidad básica de información y asume que el documento contiene una opinión principal expresada por su autor.

- Nivel de oración SA: este nivel pretende clasificar el sentimiento expresado a nivel de cada oración.

- Nivel de aspecto SA: pretende clasificar el sentimiento con respecto a los aspectos específicos de las entidades. 
La minería de opiniones es importante porque tiene aplicaciones en diferentes aéreas del conocimiento. En la educación puede ser usada para recolectar opiniones de los estudiantes sobre los temas o las estrategias de aprendizaje y esta información servirá para realizar el análisis de sentimientos y su resultado podrá ser utilizado para mejorar los materiales del curso, las estrategias de enseñanza, la personalización de contenidos, entre otros, así como también pueden ser utilizados dentro de un STI.

El análisis de sentimientos se ha utilizado en el ámbito educativo en diversos trabajos como se presenta a continuación.

Altrabsheh, Gaber, y Cocea [9] presentan SA-E, donde realizan análisis de sentimientos usando frases de Twitter para observar la retroalimentación de los estudiantes. En SA-E los estudiantes utilizaron Twitter para expresar sus opiniones sobre el material de clase, las opiniones de los estudiantes fueron utilizadas por el profesor para alterar el estilo de enseñanza de acuerdo a los resultados.

Facebook contiene un método de análisis de sentimientos que puede ser utilizado para el aprendizaje electrónico (e-learning). Los mensajes escritos por los usuarios, se usan para extraer información sobre la polaridad sentimental del mensaje (positiva, neutra o negativa), modelar la polaridad del sentimiento habitual en los usuarios y detectar cambios emocionales significativos. SentBuk [10], apoya a la detección del cambio emocional, el hallazgo emocional del amigo, la clasificación del usuario de acuerdo a sus mensajes y las estadísticas, entre otros.

El método de clasificación implementado en SentBuk sigue un enfoque híbrido, es decir, combina técnicas basadas en el léxico y la máquina. Los resultados obtenidos a través de este enfoque muestran una precisión de $83.27 \%$ en el análisis de sentimiento en Facebook. Algunas de las ventajas de e-learning es que ayuda al usuario a obtener la información sobre los sentimientos de los usuarios, de manera que esta información puede ser utilizada por sistemas adaptativos de e-learning para apoyar el aprendizaje personalizado, considerando el estado emocional del usuario y así dar una recomendación de algunas de las actividades más adecuadas para ser abordadas en el momento. Además, los sentimientos de los estudiantes hacia un curso, pueden servir como retroalimentación para los profesores ya que pueden cambiar su estrategia de enseñanza, en los Sistemas Tutores Inteligentes es posible personalizar la instrucción o mejorar el material presentado.

Por otra parte, Rowe [11] ofrece un panorama de algunos acontecimientos en el ámbito de las emociones de los estudiantes en relación con la retroalimentación, basada en de la psicología social y la educación. Altrabsheh, Cocea, and Fallahkhair [12] examinaron diferentes métodos para aprender el sentimiento de la retroalimentación de los estudiantes. Munezero, Montero, Mozgovoy, \& Sutinen [13], presentan un sistema funcional para analizar y visualizar las emociones de los estudiantes, expresadas en los diarios de aprendizaje, que son instrumentos donde los estudiantes escriben sus reflexiones sobre su experiencia de aprendizaje.

\section{Análisis de sentimientos}

En esta sección se presenta brevemente el Sistema de Evaluación de Recursos Educativos, el cual se utilizó para la creación del corpus de opiniones textuales en español para el área de programación. Además, se describe el módulo de análisis de 


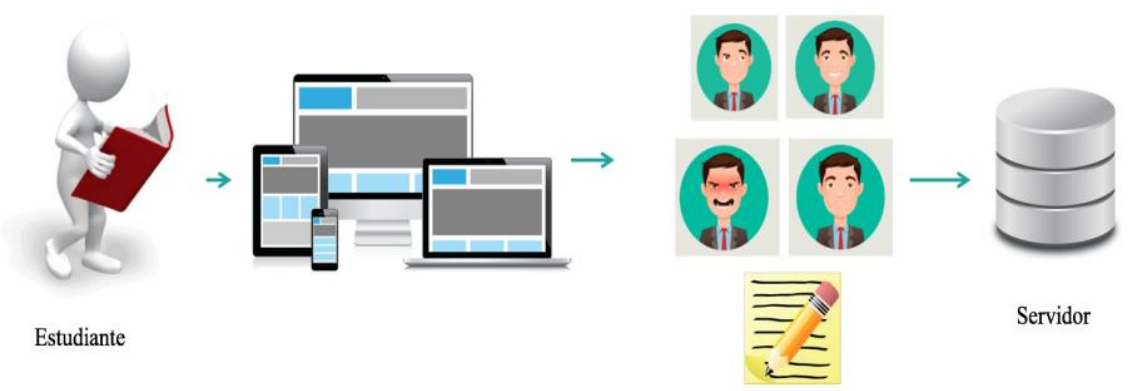

Fig. 1. Funcionamiento de Sistema de Evaluación de Recursos Educativos.

sentimientos que utiliza el corpus de opiniones para entrenamiento. Este módulo realiza la clasificación de las opiniones, esto es determinan la polaridad positiva, negativa o neutral y obtiene la emoción relacionada al aprendizaje expresada en la frase.

\subsection{Sistema de Evaluación de Recursos Educativos (SERE)}

SERE fue implementado para generar un corpus de opiniones en español enfocadas en el aprendizaje. Este sistema es una aplicación Web que fue desarrollada para la plataforma .NET en Visual Studio 2013, usando la base de datos SQL Server 2008 R2 para almacenar la información de los diferentes artefactos utilizados en el ambiente educativo, como son: cursos, recursos educativos, objetos de aprendizaje, usuarios, y opiniones emitidas por los estudiantes, etc.

SERE fue diseñado para realizar la interacción con estudiantes con el objetivo de permitir que expresen sus opiniones y comentarios libremente acerca de los recursos educativos u objetos de aprendizaje consultados sobre los temas de un curso.

En la Fig. 1 se muestra como el estudiante puede interactuar con el sistema usando diferentes dispositivos con acceso a Internet como son: Tablet, Smartphone, Laptop, o PC.

El usuario ingresa a SERE, usando su cuenta y clave de usuario, a través de la URL (http://posgradoitc.ddns.net:8000/) donde se alberga el sistema.

El usuario selecciona el curso y el sistema despliega la lista de temas y los recursos educativos disponibles, permitiendo que el usuario acceda al objeto de aprendizaje que le interesa. Posteriormente, el usuario debe escribir una opinión sobre el OA, la cual se etiqueta con la emoción que el estudiante expresa en ese momento. Cada una de las opiniones de los estudiantes son registradas en una base de datos que contiene diferentes campos como: datos del estudiante, el tema seleccionado del curso, la opinión y evaluación ingresada por el estudiante y por último la fecha y hora en que realizó la opinión. 


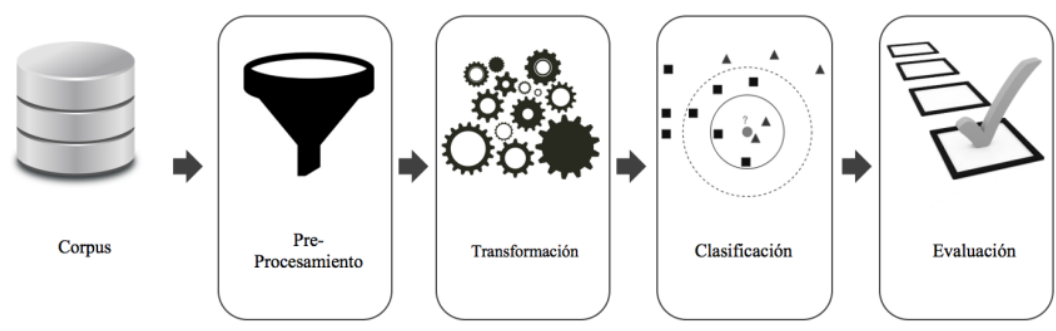

Fig. 2. Proceso del Módulo de Análisis de Sentimientos.

\subsection{Módulo de análisis de sentimientos (MAS)}

El módulo de análisis de sentimientos se encarga de determinar la polaridad (positiva, neutral o negativa) o la emoción centrada en el aprendizaje (aburrido, frustrado, emocionado y comprometido) de una frase, oración o documento.

Anteriormente el módulo de análisis de sentimiento [14] determinaba solamente la polaridad del texto (positiva, neutral o negativa), este módulo se adaptó para reconocer las emociones centradas en el aprendizaje agregando las etiquetas: aburrido, frustrado, comprometido y emocionado. El módulo de análisis de sentimientos se desarrolló usando el clasificador Bernoulli Naive Bayes porque es de los clasificadores más simples y comúnmente utilizados sin embargo utiliza otros tipos de clasificadores como son: Multinomial Naive Bayes, Support Vector Machine, Linear Support Vector Machine, Stochastic Gradient Descent Classifier, and K-Nearest Neighbors.

Para probar el funcionamiento del módulo de análisis de sentimientos, se utilizó un corpus de frases etiquetadas con su respectiva emoción centrada en el aprendizaje las cuales fueron recolectadas de diversas fuentes como: SERE [17], Twitter, plataformas educativas y del corpus TASS [15].

La Fig. 2 muestra el proceso que utiliza el módulo de análisis de sentimiento donde se aprecia que el Corpus de frases es la entrada al proceso que consta de 4 pasos.

Pasos del proceso para análisis de sentimientos:

1. Pre-procesamiento: normaliza las frases contenidas en el corpus. Los pasos de pre-procesamiento son:

a) Slang terms: traduce la jerga y emoticones a su equivalente en texto.

b) Tokenizador: separa las sentencias en palabras removiendo puntos y signos.

c) Stop-words: remueve las palabras innecesarias.

d) Stemming: reduce las palabras a su palabra raíz.

2. Transformación: se genera una matriz TF-IDF que calcula la frecuencia de un término (número de veces que aparece un término dado en un documento o conjunto de datos) y la frecuencia inversa de documento (número de documentos en los que aparece un término dado) para cada palabra en el corpus. Esto se conoce como extracción de características de ponderación.

3. Clasificación: define una función para predecir la etiqueta ingresada como entrada. 
Uso de analizador de emociones en sistemas educativos inteligentes

Tabla 1. Opiniones en el Corpus de frases etiquetadas con emoción centrada en el aprendizaje.

\begin{tabular}{llcc}
\hline Clave & \multicolumn{1}{c}{ Opinión en Español } & Formato & Evaluación \\
\hline E-15 & Me gusto bastante el vídeo & Video & Comprometido \\
\hline E-64 & $\begin{array}{l}\text { No me gusto que las voces fueran de España, además } \\
\text { los gráficos de la animación están algo feos. }\end{array}$ & Video & Frustrado \\
\hline E-70 & $\begin{array}{l}\text { El video es bueno aunque creo que le falto profundizar } \\
\text { mas }\end{array}$ & Video & Neutral \\
\hline E-29 & Vaya, es algo complejo & Video & Frustrado \\
\hline E-67 & Quizás con un ejemplo quedaría más claro. & $\begin{array}{c}\text { Imagen- } \\
\text { Texto }\end{array}$ & Aburrido \\
\hline E-71 & $\begin{array}{l}\text { Hubiese sido mejor poner una tabla con sus diferencias } \\
\text { y así compararlas y sea más diverso. }\end{array}$ & $\begin{array}{c}\text { Imagen- } \\
\text { Texto }\end{array}$ & Emocionado \\
\hline
\end{tabular}

4. Evaluación: evalúa el modelo de aprendizaje máquina para predecir la polaridad (positivo, negativo) de un texto de entrada; esto nos ayuda a encontrar un modelo confiable.

\section{Resultados}

En esta sección, se presentan los resultados obtenidos en los experimentos realizados con el clasificador de frases.

\subsection{Evaluación del sistema de evaluación de recursos educativos}

El primer experimento fue realizado en Agosto 2017, y participaron 53 estudiantes (45 hombres y 8 mujeres) de la carrera de Ingeniería en Sistemas Computacionales del Instituto Tecnológico de Culiacán. Los estudiantes usaron SERE, con el curso de Fundamentos de Programación y los recursos educativos de la unidad 1. Después de estudiar cada subtema en formato de texto e imágenes o multimedia (video), el estudiante registró su opinión con un texto de longitud de 15 a 255 caracteres respecto al contenido del tema estudiado, además, seleccionó una etiqueta para su opinión usando un emoticón que representaba su estado emocional: aburrido, frustrado, neutral, emocionado o comprometido. En la Tabla 1 se muestran algunos ejemplos de las opiniones registradas por los estudiantes que participaron en el estudio.

Con la información obtenida en esta evaluación de SERE, primero se validó con un grupo de profesores que las opiniones de los estudiantes tuvieran relación con la emoción capturada con el emoticón que tenía asociado. Se encontró un 5\% de incongruencias y se realizaron las correcciones correspondientes. En este proceso también se detectó que el $20 \%$ opiniones escritas eran definiciones de términos básicos tal como algoritmo, computadora, entre otras, por lo que esas opiniones se consideraron con la emoción neutral. Posteriormente se utilizó el Módulo Analizador de Sentimiento para validar las coincidencias con el contenido emocional de los textos, considerando frustrado y aburrido como una emoción negativa y las etiquetas: neutral, emocionado y comprometido como una emoción positiva dado que el Módulo Analizador solo reconocía textos etiquetados como positivos o negativo. 


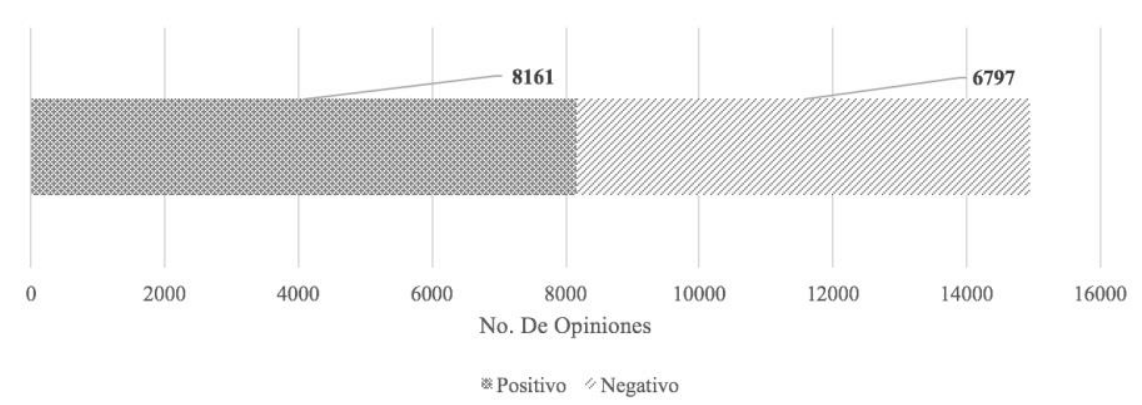

Fig. 3. Distribución del corpus por Polaridad.

\subsection{Evaluación del modelo de aprendizaje máquina}

Una serie de métricas se utilizan para estimar la calidad del algoritmo de clasificación que es parte del modelo de aprendizaje propuesto. En este caso, se usó el método más simple para calcular la efectividad de un clasificador, que es la medida de precisión. Esta calcula el porcentaje de documentos de texto correctamente clasificados sobre el total de documentos a clasificar. Para obtener este valor, se aplicó una técnica de validación cruzada con un conjunto de datos en español con un $90 \%$ para los datos de entrenamiento y un $10 \%$ para los datos de prueba.

\subsection{Corpus de opiniones etiquetados con polaridad}

El corpus de frases generado con SERE, contiene tanto etiquetas de polaridad (positivo y negativo) como etiquetas de emociones centradas en el aprendizaje (frustrado, aburrido, comprometido y emocionado).

Actualmente el corpus contiene 14,958 frases, la Fig. 3 muestra la distribución del corpus por polaridad, el cual contiene 8,161 opiniones positivas y 6,797 negativas.

Para el análisis de sentimientos usando el corpus generado con SERE, se utilizaron los siguientes algoritmos de clasificación: Bernoulli Naïve Bayes, Multinomial Naïve Bayes, Support Vector Machine, Linear Support Vector Machine, Stochastic Gradient Descent, and K-Nearest Neighbors (KNN). El clasificador con el puntaje más alto fue Bernoulli Naïve Bayes con una exactitud (accuracy) del 83.56\%. En la tabla 2 se muestra los valores obtenidos utilizado el clasificador Bernoulli NB.

\subsubsection{Corpus de opiniones etiquetadas con emociones centradas en el aprendizaje}

Uno de los principales objetivos del SERE fue recopilar opiniones de los estudiantes para generar un nuevo corpus de opiniones en español para reconocer emociones centradas en el aprendizaje (frustrado, aburrido, emocionado y comprometido) con la finalidad de mejorar el Módulo Analizador de Sentimiento que solo reconoce polaridad, esto es, dos estados emocionales: positivo y negativo. En la Fig. 4 se muestra la distribución de las opiniones recolectadas de septiembre 2017 a marzo 2018. 
Tabla 2. Valores obtenidos usando el clasificador Bernoulli Naïve Bayes.

\begin{tabular}{ccccc}
\hline & Precisión & Recall & F1-score & Accuracy \\
\hline Avg/ total & 0.84 & 0.84 & 0.84 & 0.835 \\
\hline
\end{tabular}

Tabla 3. Valores obtenidos usando el clasificador Bernoulli Naïve Bayes.

\begin{tabular}{ccccc}
\hline & Precisión & Recall & F1-score & Accuracy \\
\hline Avg/ total & 0.58 & 0.57 & 0.54 & 0.574 \\
\hline
\end{tabular}

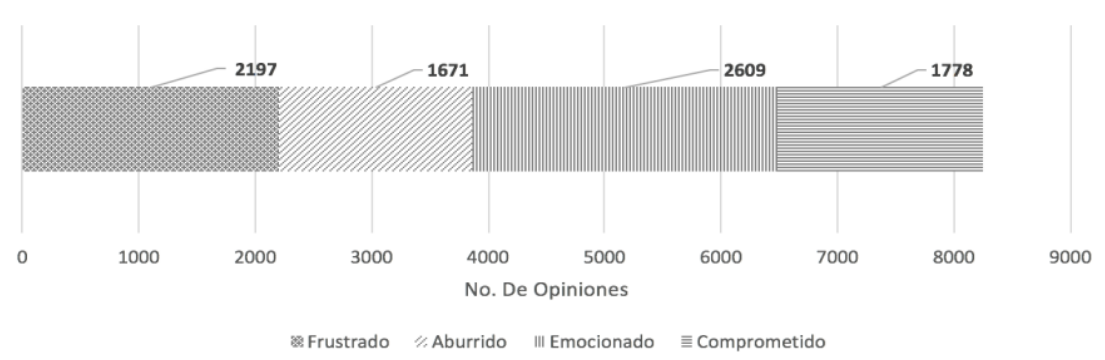

Fig. 4. Distribución del corpus por Emociones Centradas en el Aprendizaje.

El corpus de opiniones etiquetadas con emociones centradas en el aprendizaje se utilizó para entrenar el algoritmo de clasificación Bernoulli Naïve Bayes obteniendo una accuracy de $57.40 \%$. En la Tabla 3 se muestran los resultados obtenidos.

\section{Conclusiones}

En este trabajo se generó un corpus de emociones basadas en el aprendizaje utilizando el sistema SERE el cual contiene 9,000 opiniones textuales y cada una de ellas esta etiquetada con una emoción relacionada con el aprendizaje. Además, se trabajó con un módulo de análisis de sentimiento en el cual actualmente trabaja con dos diferentes formas de clasificar una frase de texto.

La primera es utilizando el corpus de emociones centradas en el aprendizaje la cual tiene una precisión de $57.40 \%$ utilizando el algoritmo de clasificación Bernoulli Naïve Bayes. La segunda es utilizando el corpus etiquetado con polaridad en el cual se utilizado diferentes algoritmos de clasificación sin embargo la que obtiene mejor precisión es Bernoulli Naïve Bayes con $76.77 \%$.

Por otra parte, el corpus será utilizado posteriormente para que el administrador de un Ambiente Inteligente de Aprendizaje tome decisiones acerca de la pertinencia de los recursos educativos que contiene el sistema y proponga cambios o mejoras a los mismos. Esto ayudará a que los Sistemas Tutores Inteligentes detecten emociones a través de texto y realicen de manera más eficiente el proceso de enseñanza con los estudiantes, ajustando el contenido a las necesidades particulares de cada uno de ellos.

Para trabajos futuros, se está considerando la inclusión de ejercicios para desarrollo de programas Java en el sistema SERE, esto permitirá además crear un banco de 
problemas para el aprendizaje de la programación de computadoras, así como ampliar el corpus de opiniones en esta área del conocimiento.

\section{Referencias}

1. D’Mello, S., Jackson, T., Craig, S., Morgan, B., Chipman, P., White, H., Graesser, A.: AutoTutor detects and responds to learners affective and cognitive states. In: Workshop on emotional and cognitive issues at the international conference on intelligent tutoring systems, pp. 306-308 (2008)

2. Ekman, P.: An argument for basic emotions. Cognition and Emotion, 6, pp. 169-200 (1992)

3. Baker, R.S.J.d., D'Mello, S.K., Rodrigo, M.M.T., Graesser, A.: Better to be frustrated than bored: The incidence, persistence, and impact of learners' cognitive affective states during interactions with three different computer-based learning environments. Int. J. Hum.Comput. Stud., 68, pp. 223-241 (2010)

4. D'Mello, S.K., Graesser, A.: Dynamics of affective states during complex learning. Learning and Instruction, 22(2), pp.145-157 (2012)

5. Pekrun, R.: The impact of emotions on learning and achievement: Towards a theory of cognitive/motivational mediators. Applied Psychology, 41(4), pp. 359-376 (1992)

6. Pekrun, R., Goetz, T., Titz, W., Perry, R.P.: Academic emotions in students' self-regulated learning and achievement: A program of qualitative and quantitative research. Educational psychologist, 37(2), pp. 91-105 (2002)

7. Feldman, R.: Techniques and applications for sentiment analysis. Communications of the ACM, 56(4), pp. 82-89 (2013)

8. Medhat, W., Hassan, A., Korashy, H.: Sentiment analysis algorithms and applications: A survey. Ain Shams Engineering Journal, 5(4), pp. 1093-1113 (2014)

9. Altrabsheh, N., Gaber, M., Cocea, M.: SA-E: Sentiment Analysis for Education. Frontiers in Artificial Intelligence and Applications, 255 (2013)

10. Ortigosa, A., Martín, J.M., Carro, R.M.: Sentiment analysis in Facebook and its application to e-learning. Comput. Hum. Behav., 31, pp. 527-541 (2014)

11. Rowe, A.D.: Feelings About Feedback: The Role of Emotions in Assessment for Learning. In: Scaling up Assessment for Learning in Higher Education, Carless, D., Bridges, S.M., Chan, C.K.Y., Glofcheski, R., Eds. Singapore: Springer Singapore, pp. 159-172 (2017)

12. Altrabsheh, N., Cocea, M., Fallahkhair, S.: Learning Sentiment from Students' Feedback for Real-Time Interventions in Classrooms. In: Adaptive and Intelligent Systems: Third International Conference, (ICAIS'14), Proceedings, A. Bouchachia, Cham: Springer International Publishing, pp. 40-49 (2014)

13. Munezero, M., Montero, C.S., Mozgovoy, M., Sutinen, E.: Exploiting sentiment analysis to track emotions in students' learning diaries. In: Proceedings of the 13th Koli Calling International Conference on Computing Education Research, pp. 145-152 (2013)

14. Barrón-Estrada, M.L., Zatarain-Cabada, R., Oramas-Bustillos, R., González-Hernández, F.: Sentiment Analysis. In: An Affective Intelligent Tutoring System, IEEE 17th International Conference on Advanced Learning Technologies (ICALT), Timisoara, pp. 394-397 (2017)

15. Villena-Román, J., Mártinez-Cámara, E., Lana-Serrano, S., González-Cristóbal, J.C.: TASS-Worlshop on Sentiment Analysis at SEPLN TASS. Taller de Análisis de Sentimientos en la SELPLN, pp. 37-44 (2013)

16. Tellez, E.S., Miranda-Jiménez, S., Graff, M., Moctezuma, D., Siordia, O.S., Villaseñor, E. A.: A case study of Spanish text transformations for twitter sentiment analysis. Expert Systems with Applications, pp. 457-471 (2017)

17. Barrón-Estrada, M.L., Zataraín-Cabada, R., Oramas-Bustillos, R., Ramírez-Ávila, S.L.: Building a Corpus of Phrases Related to Learning for Sentiment Analysis. Research in Computing Science, 146, pp. 17-26 (2017) 